(IDDF2021-ABS-0054 Figure 5B) to pro-inflammatory and pro-oncogenic gut microecological environment.

Conclusions Our population-based studies showed appendectomy significantly increases the risk of CRC development. Appendectomy-induced gut dysbiosis and related functional pathways contribute as least in part to the appendectomy-associated CRC risk.

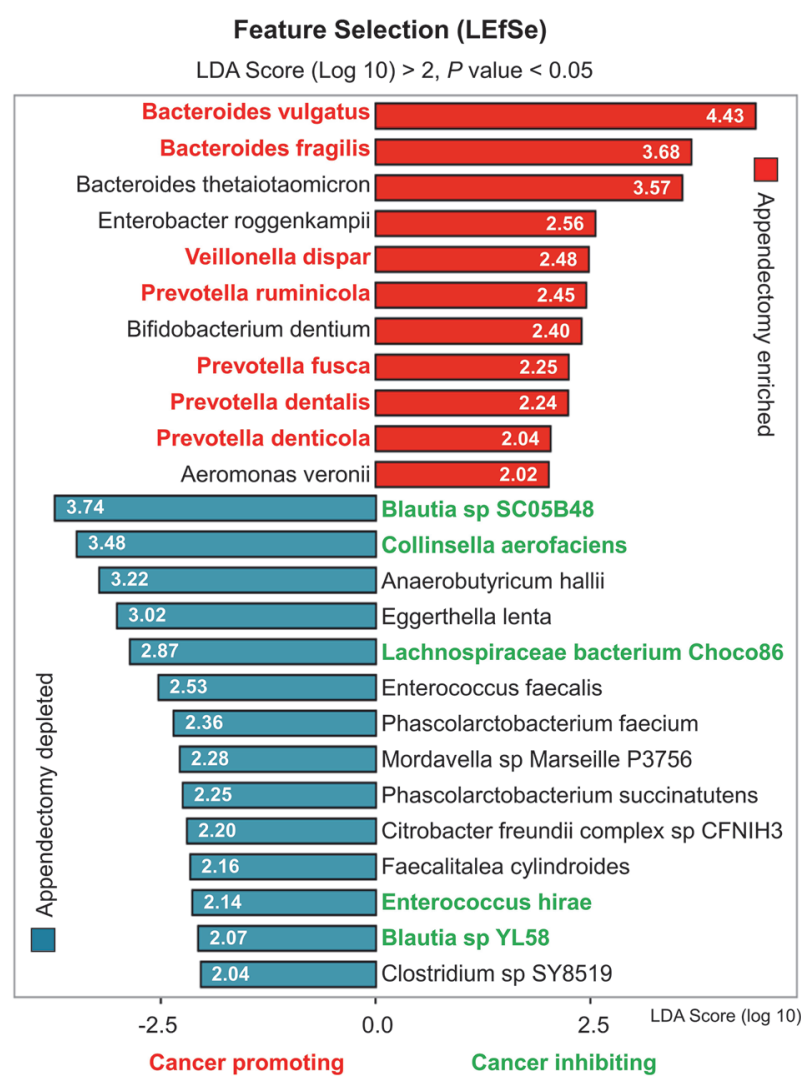

Abstract IDDF2021-ABS-0054 Figure 4

A

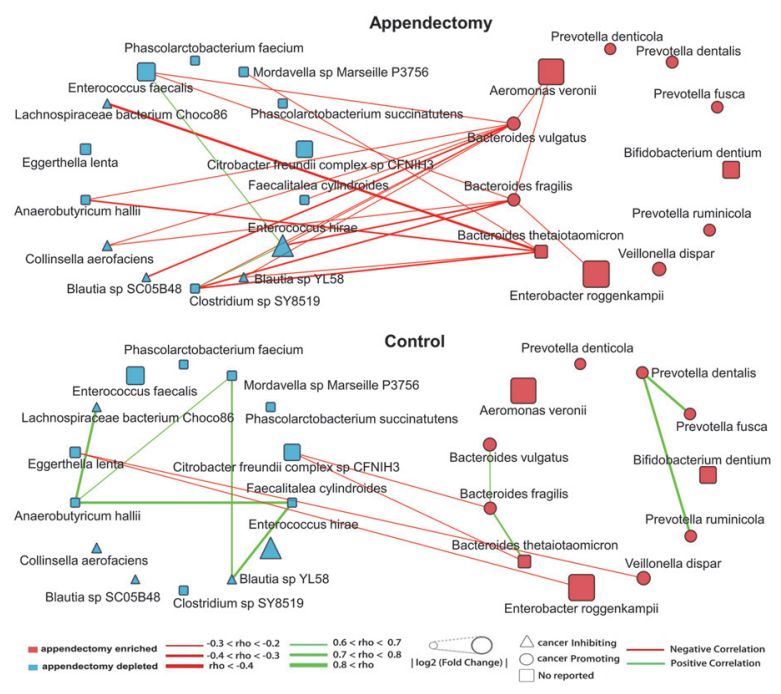

IDDF2021-ABS-0061

TM9SF4 AMELIORATES INFLAMMATORY
BOWEL DISEASE BY ALLEVIATING
INFLAMMATION AND ER STRESS IN
COLONIC EPITHELIAL CELLS AND
MACROPHAGES

${ }^{1}$ Mingxu Xie*, ${ }^{1}$ Hongyan Yu, ${ }^{1}$ Wing Hung Ko, ${ }^{2}$ Wing Yan Mak, 'Xiaogiang Yao. ${ }^{1}$ School of Biomedical Sciences, The Chinese University of Hong Kong, Hong Kong; ${ }^{2}$ Department of Medicine and Therapeutics, The Chinese University of Hong Kong, Hong Kong

\subsection{6/gutjnl-2021-IDDF.25}

Background Inflammatory bowel disease (IBD) is a result of excess inflammation and inappropriate immune response to environmental changes and the gut microbiota. The search of genome-wide association study (GWAS) database identified a remarkable correlation between TM9SF4 gene and IBD, with p-value $=6 \times 10^{10}$. In the previous studies, TM9SF4 was found to be highly expressed in macrophages and intestinal epithelial cells (IECs), promoting phagocytosis and innate immune response. Besides, TM9SF4 reduced cell stress to protect cells from apoptotic deaths. Therefore, it is likely that TM9SF4 might exert protective roles in IBD via its actions of reducing inflammation and alleviating ER stress in both macrophages and IECs.

Methods Expression levels of TM9SF4 were compared between actively inflamed and non-inflamed regions of colons from IBD patients by immunohistochemistry and western blotting. Dextran sulfate sodium (DSS)-induced colitis was established in wild-type (WT) and TM9SF4 knockout (KO) mice. Bone marrow transplantation was used to elucidate the contribution of myeloid cells and IECs in disease progression. Intestinal permeability, IECs stress and deaths, macrophage phagocytosis functions and polarization were measured with in vitro and in vivo techniques.

Results Decreased expression of TM9SF4 was observed in inflamed regions of colon tissues from patients with IBD. Patients with a higher Mayo score showed lower expression of TM9SF4 in their colons. In animal studies, knockout of TM9SF4 aggravated DSS-induced colitis, which could be attributed to TM9SF4 deficiency in bone marrow-derived hematopoietic cells as well as in colonic epithelial cells. For

B Functional prediction in appendectomy and control groups

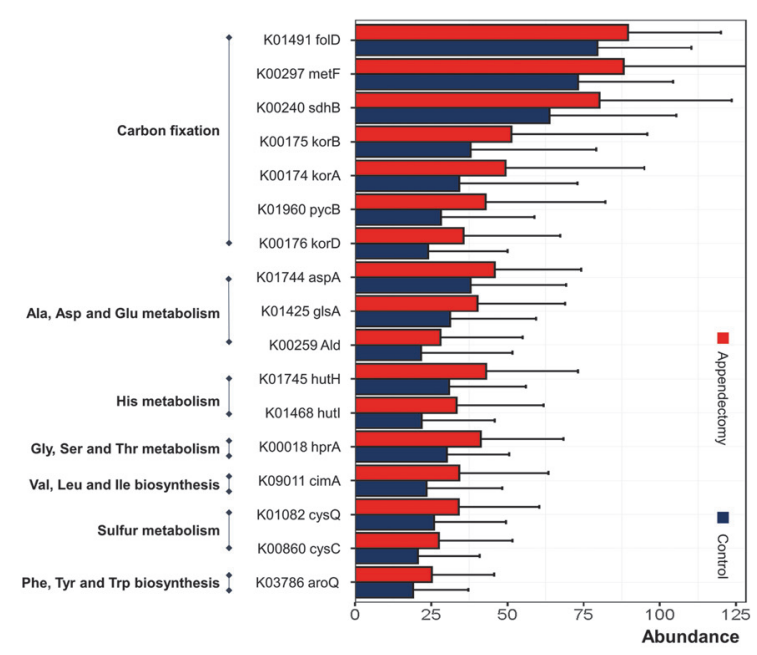


IECs, loss of TM9SF4 impaired epithelial barrier functions by promoting inflammation, elevating ER stress, oxidative stress and consequently contributing to apoptotic deaths. For macrophages, more colonic infiltration of macrophages was observed in TM9SF4 KO mice than in WT mice. Besides, TM9SF4 facilitated clearance of apoptotic cells by colonic macrophages, enhanced monocytes polarization into proinflammatory M1 macrophages while suppressed M2 macrophages polarization in vitro and in vivo.

Conclusions Our studies suggested that TM9SF4 in intestinal epithelial cells and macrophages may act in coordinated ways to exert its anti-inflammatory role in IBD.

\section{IDDF2021-ABS-0065 THE TUMOR IMMUNOLOGICAL CHARACTERIZATIONS OF COLON ADENOCARCINOMA REVEAL CLINICALLY RELEVANT POPULATION-SPECIFIC DIFFERENCES}

Midie Xu*, Jinjia Chang, Cong Tan, Xin Wang, Weiwei Weng, Shujuan Ni, Meng Zhang, Lei Wang, Wenhua Li, Dan Huang, Weiqi Sheng. Fudan University Shanghai Cancer Center, China

10.1136/gutjnl-2021-IDDF.26
Background Immunomodulation has become a topical area of interest in many tumors, being colon adenocarcinoma (COAD), not an exception. Considering the molecular heterogeneity of COADs and their non-immunogenic character, immunotherapy only showed a viable role in a certain subset of COADs. This study aimed to determine immune subtypes (ISs) of COADs for the selection of suitable patients from an extremely heterogeneous population.

Methods Gene expression profiles and corresponding clinical information were collected from TCGA and GEO databases, respectively. Consensus clustering analysis was performed to identify the ISs. Immunogenomics methods were integrated to characterize the immune environment of each IS subtype. Linear discriminant analysis was conducted to establish an immune subtyping characteristic index (ISCI) for IS classification of COADs. Co-expression network analysis was used for detected hub genes. DAVID was used for functional annotation.

Results Based on 17 prognostic immune characteristics, COADs were stratified into three ISs characterized by differential molecular, cellular and clinical features. Patients with the IS1 tumor had immune 'hot' and immunosuppressive phenotype, IS3 tumor had immune 'hot' phenotype, whereas those with the IS2 tumor had immune 'cold' phenotype. Patients with the IS1 tumor had the worst disease-free survival
A

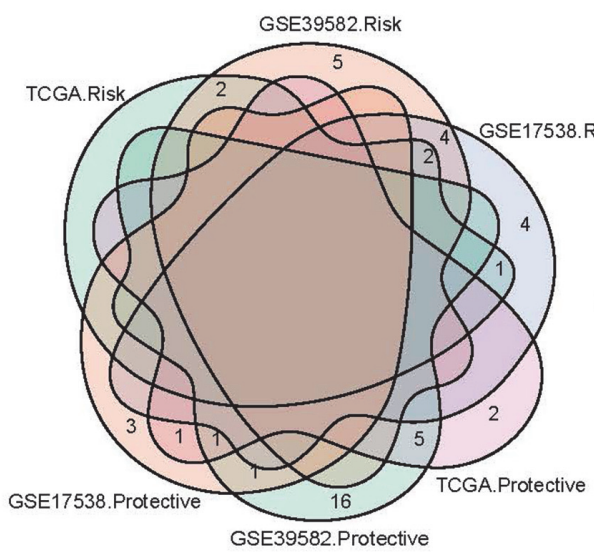

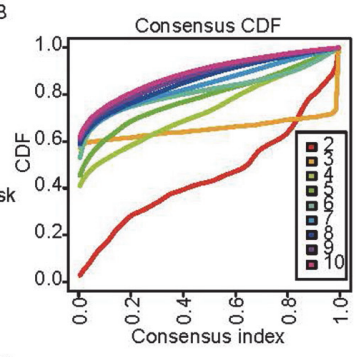

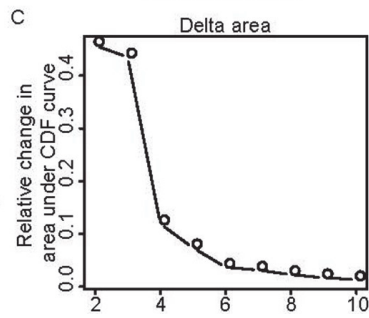

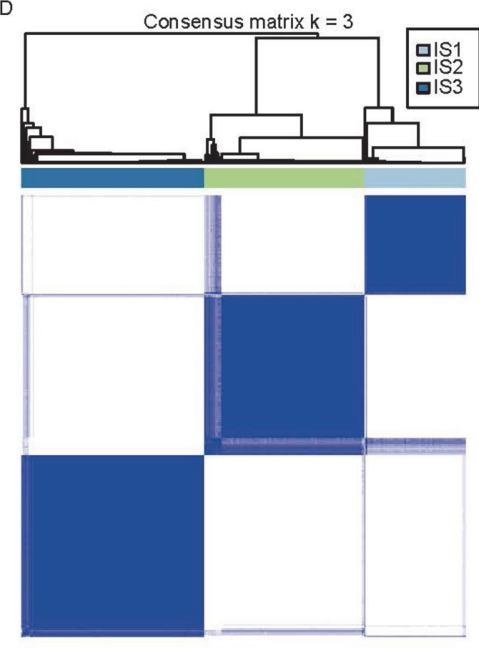

\section{Abstract IDDF2021-ABS-0065 Figure 1}

E

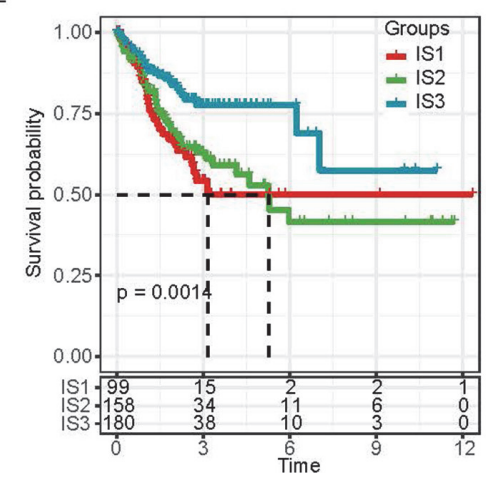

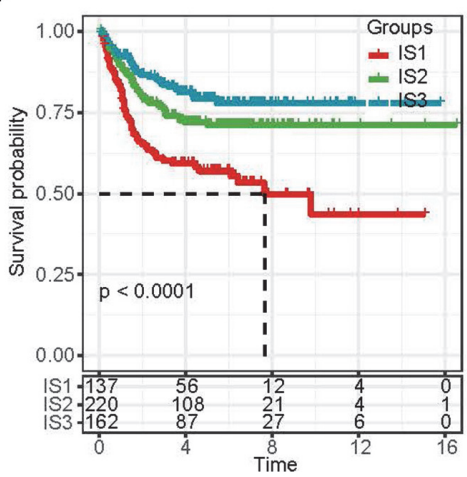

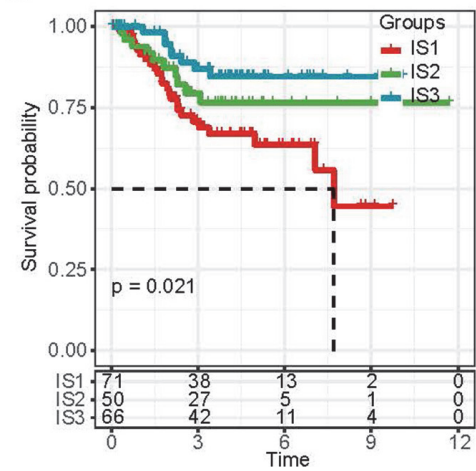

